\title{
Fully Integrated K-Band Active Bandpass Filter In GPDK 90nm CMOS Technology
}

\author{
Md. Jamil Uddin \\ Electrical and Electronic Engineering \\ Southern University Bangladesh \\ 739/A, Mehedibag Road, Chittagong, \\ Bangladesh. \\ email: mzuddin62@gmail.com
}

\author{
Hadaate Ullah \\ Electrical and Electronic Engineering \\ Southern University Bangladesh \\ 739/A, Mehedibag Road, Chittagong, \\ Bangladesh. \\ email: hadaate@southern.edu.bd
}

\author{
Mohammad Arif Sobhan Bhuiyan \\ Electrical and Electronics Engineering \\ Xiamen University Malaysia \\ Jalan Sunsuria, Bandar Sunsuria, \\ 43900 Sepang, Selangor, Malaysia. \\ email: arifsobhan.bhuiyan@xmu.edu.my
}

\begin{abstract}
The bandpass filter is one of the essential blocks of every modern RF transceiver. Performance of the transceiver greatly depends on the performance of the bandpass filter. A bandpass filter designed with passive inductors suffers from some drawbacks like large chip size, low-quality factor, less tenability etc. To prevail over these constraints, an active inductor-based bandpass filter circuit has been designed in GPDK-90nm CMOS technology utilizing cadence virtuoso environment. The simulation result shows that the active inductor-based bandpass filter circuit design achieves a gain of 6.79dB, a bandwidth of $5.05 \mathrm{GHz}$ and a noise figure of $3.10 \mathrm{~dB}$. The circuit dissipates only $3.55 \mathrm{~mW}$ power for its operation from a single $1.5 \mathrm{~V}$ DC supply. By avoiding bulky inductor in the design helped to attain a very small chip area of $127.704 \mu \mathrm{m}^{2}$.
\end{abstract} $R F$.

Keywords-active inductor, band-pass filter, CMOS, K-band,

\section{INTRODUCTION}

Inductors, in both passive or active form, play an important role in analogue components of typical radio frequency (RF) devices such as filters, amplifiers, oscillators, mixers, phase shifters, impedance matching circuitries, etc. of applications including mobile phones (GSM 900/1800), Global Position System (GPS), television broadcasting, Wireless Local Area Network (WLAN), Bluetooth, Radio Frequency Identification (RFID), Zigbee and many more [1-2]. Generally, RF band-pass filters are realized with integrated inductors. Unfortunately, the parasitic effects of losses in substrate and coupling capacitance demean the overall performance, especially for silicon chips. Moreover, the consequences like large chip area, low-quality factor, fixed tuning etc. are making passive inductors less interested to RFIC designers [3].

In order to overcome the limitations, active inductors composed of MOS transistors are initiated. They can be managed to tune accordingly to overcome the effects of PVT variations. Moreover, they can be designed to exhibit higher inductance and quality factor by taking only up to $10 \%$ of the area compared to its passive counterpart [4]. As a result, despite rapid improvements in integrated circuit technologies in recent years, the usage of passive inductors is lessening day by day.
The idea of the active inductor, as shown in Fig.1, is derived from the gyrator theory [5], which is nothing but a two-port network consisting of two transconductors connected in negative feedback. The input impedance of such a circuit is given by:

$$
Z_{\text {in }}=V_{\text {in }} / I_{\text {in }}=\left(s C / G_{m 1} . G_{m 2}\right) \text {, }
$$

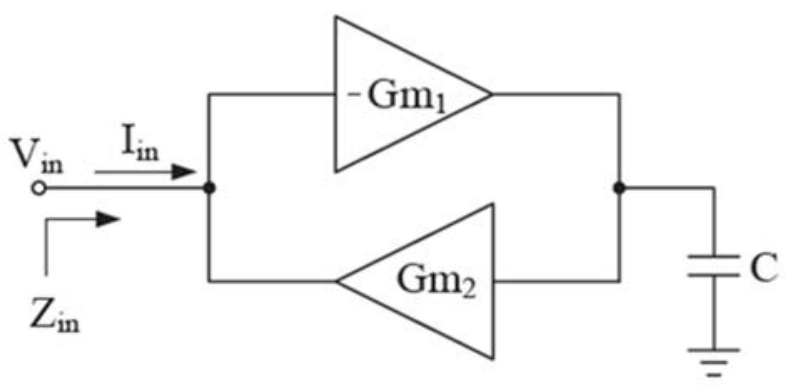

Fig.1. Concept of a typical gyrator.

The transconductors replaced by NMOS transistors can result in the simple active inductor as illustrated in Fig.2. Nevertheless, this structure exhibits few drawbacks, for instance, small inductance value, low-quality factor, and constricted tuning limit [6]. To conquer these constraints, several techniques have been introduced as illustrated in the literature [7-8].

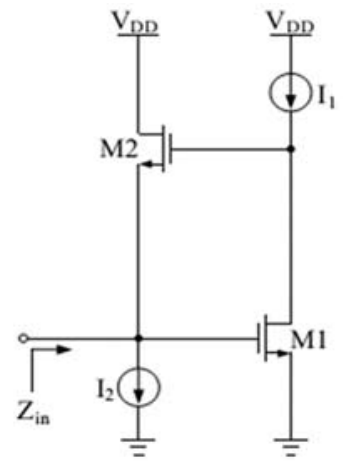

Fig.2. Basic CMOS active inductor [6] 
Continuous downscaling of CMOS technology enables the designers fabricating fully integrated and low power RF systems [9]. An RF filter is one of the performance decisive building blocks of all modern wireless transceivers. Especially in microwave frequencies, integrated inductors, as part of the filter circuit, are not recommended due to their loss and size issues. Therefore, in this research, a fully integrated active inductorbased active bandpass filter design has been proposed in GPDK 90nm CMOS technology for K-band RF devices.

\section{Filter Design}

To design an active inductor circuit for $\mathrm{GHz}$ frequencies, opamps and linearized transconductors $\left(\mathrm{G}_{\mathrm{m}}\right)$ cannot be used because of excess parasitic capacitance effect and phase shift problem. Regarding that, minimum-transistor count $\mathrm{G}_{\mathrm{M}}$ must be occupied in order to achieve the desired frequencies. An active inductor can become a second-order resonator in RF frequencies as a result of tank circuit composed of parasitic capacitances and the equivalent inductor.

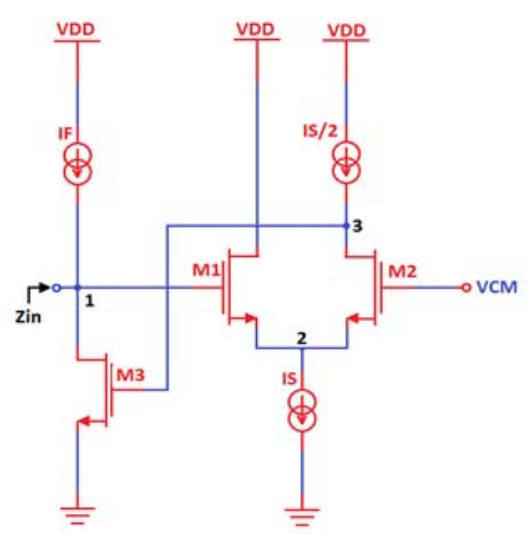

Fig.3. The simplified circuit diagram of the active inductor with all

$$
\text { NMOS signal path }
$$

An active inductor offers high impedance at the selfresonance frequency $\left(f_{0}\right)$ to build a bandpass filter. Fig. 3 shows the simplified all transistor active inductor circuit and its equivalent inductor along the parasitics is illustrated in Fig. 4. For clarity, the ideal current sources are used instead of currentmirror MOSFETs. By tracing the signal flow, the operation of the circuit can be understood.

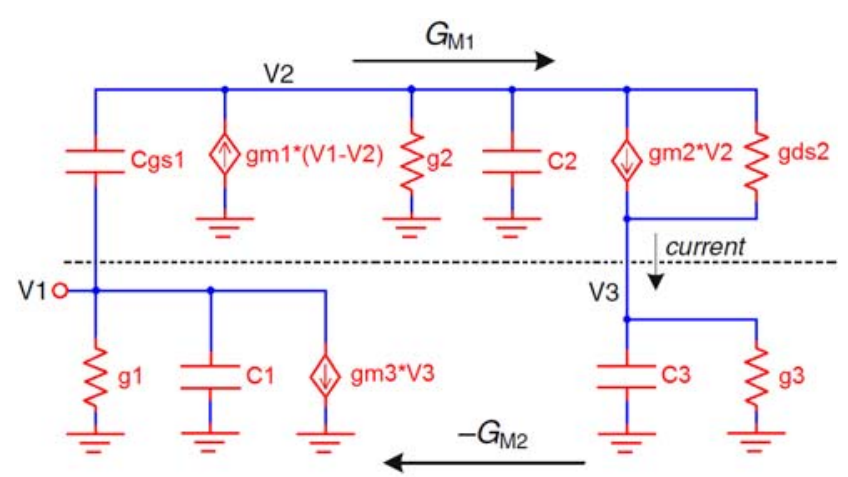

Fig.4. Small-signal equivalent circuit of the active inductor
Transistor $\mathrm{M}_{1}$ and $\mathrm{M}_{2}$ acts as a non-inverting $\mathrm{Gm}$ cell which is labeled as $\mathrm{G}_{\mathrm{M} 1}\left(=0.5 \mathrm{~g}_{\mathrm{m} 1}\right.$, if $\mathrm{g}_{\mathrm{m} 1}=\mathrm{g}_{\mathrm{m} 2}$ and $\left.\mathrm{g}_{2}=0\right)$ with the input voltage at $\mathrm{V}_{1}$ and output current at $\mathrm{V}_{3} . \mathrm{M}_{3}$ is an inverting transconductor which labeled as $-\mathrm{G}_{\mathrm{M} 2}\left(=-\mathrm{g}_{\mathrm{m} 3}\right)$ with the input voltage at $\mathrm{V}_{3}$ and output current at $\mathrm{V}_{1}$. The signal passes through NMOSs only because of their higher motilities and the circuit can be operated at higher frequencies.

The construction of the proposed BPF is given in Fig. 5. The input signal is fed to the input buffer and then it is passed to the active inductor part and lastly into an output buffer.

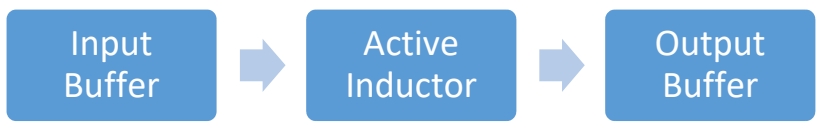

Fig.5. Block diagram of the active inductor-based BPF

The schematic circuit of the proposed bandpass filter with proper biasing and tuning components is given in Fig. 6.

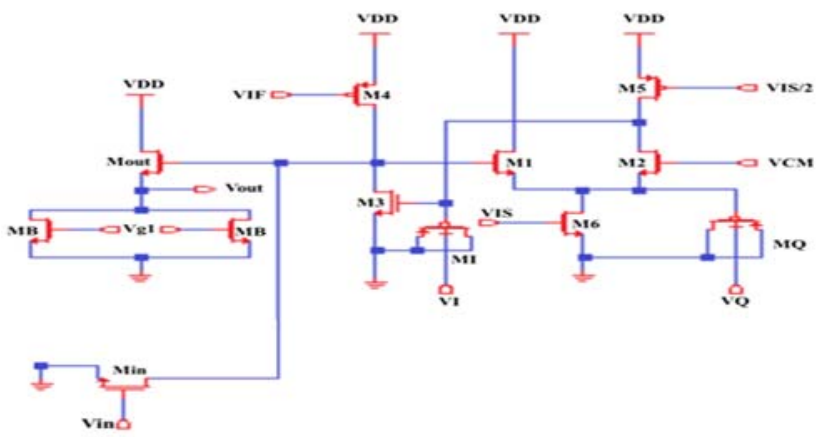

Fig. 6 Bandpass filter with. transistors Sizes (W/L in $\mu \mathrm{m})$ and bias voltages are M1,3,410/0.10, M230/0.10, M5,63/0.10, Min,I,Q,B 0.12/0.10, Mout1/0.10, VIF, IS/2,IS $=0.85 \mathrm{~V}, \mathrm{VI}=0.65 \mathrm{~V}, \mathrm{VQ}=0.7 \mathrm{~V}, \mathrm{Vg} 1,2=1 \mathrm{~V}$, $\mathrm{VCM}=0.7 \mathrm{~V}, \mathrm{VDD}=1.5 \mathrm{~V}$.

\section{RESULTS AND DISCUSSIONS}

The proposed bandpass filter circuit has been designed and simulated by cadence virtuoso tool for GPDK-90nm CMOS technology in ADE-L environment. The circuit is powered by a single $1.5 \mathrm{~V}$ supply In this research, gain, noise figure, bandwidth and quality factor have been evaluated to measure its performance.

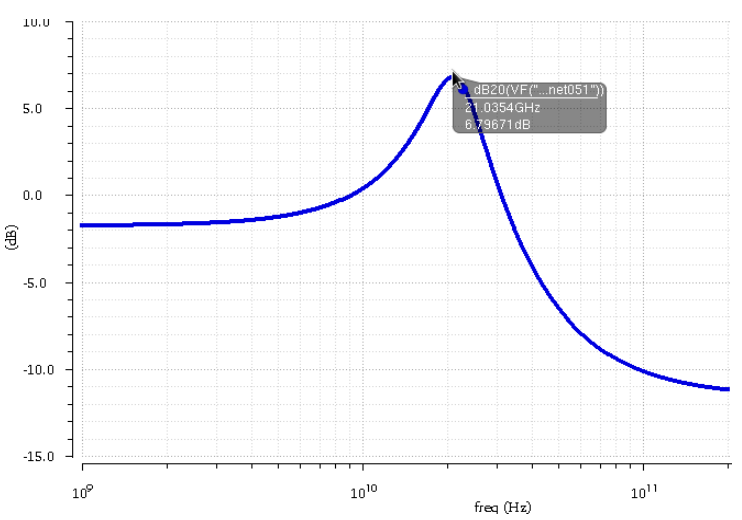

Fig. 7 AC analysis of the bandpass filter circuit 


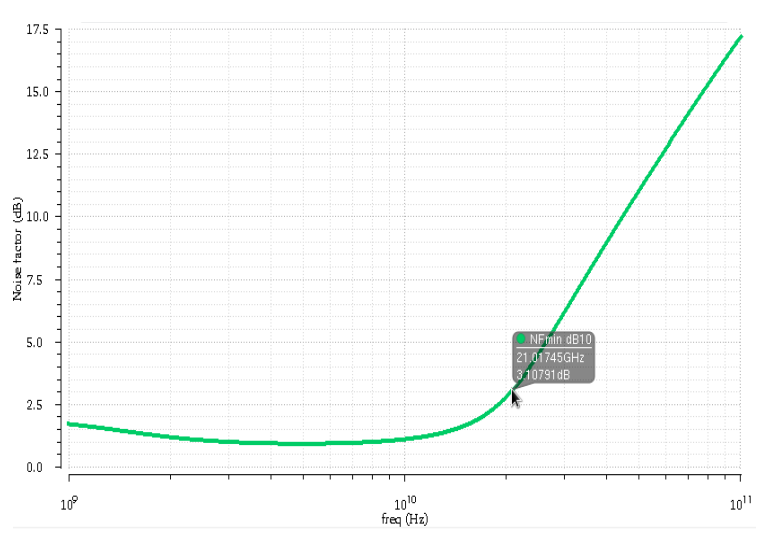

Fig. 8 Noise figure analysis of the proposed bandpass filter circuit

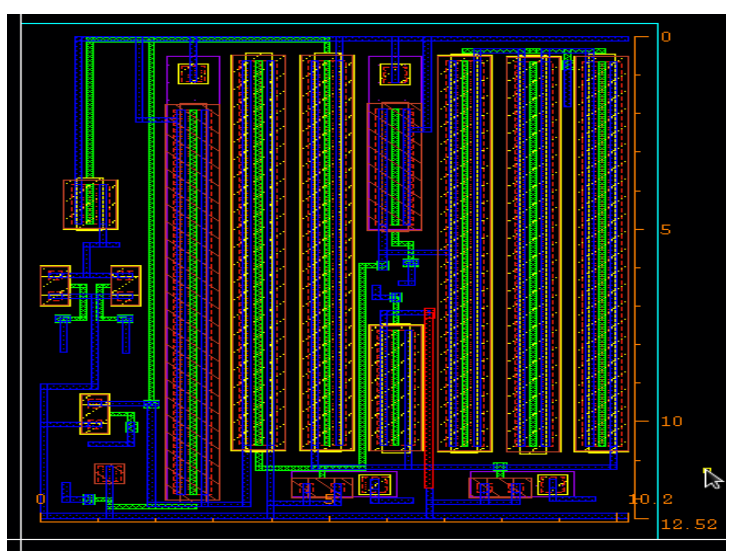

Fig. 9 Layout design for proposed bandpass filter circuit with an area of $(10.2 \times 12.52) \mu \mathrm{m}^{2}$.

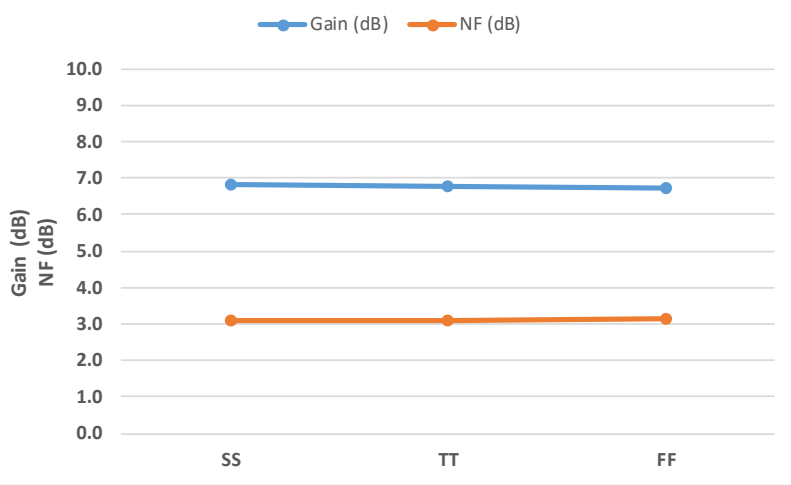

Fig. 10 FEOL corner analysis for the proposed bandpass filter.

The measured result of the proposed bandpass filter circuit is shown in Fig 7. We can observe that the proposed bandpass filter circuit have gain $6.79 \mathrm{~dB}$, can operate at the center frequency of $21 \mathrm{GHz}$ which has the value of quality factor of 4.16 and bandwidth of $5.05 \mathrm{GHz}$.
The noise figure of the proposed bandpass filter is shown in Fig. 8, which is $3.10 \mathrm{~dB}$. The total power dissipation, including the biasing and buffer circuits, equals to $3.55 \mathrm{~mW}$ at $1.5 \mathrm{~V}$ power supply. Basically, a much lower supply voltage will result in a lower power dissipation.

TABLE I. SUMMERY OF THE BANDPASS FILTER

\begin{tabular}{|c|c|}
\hline Parameters & Values \\
\hline Center frequency $(\mathrm{GHz})$ & 21 \\
\hline Gain(dB) & 6.79 \\
\hline Bandwidth $(\mathrm{GHz})$ & 5.05 \\
\hline Quality factor & 4.16 \\
\hline Noise Figure(dB) & 3.10 \\
\hline Power Supply(V) & 1.5 \\
\hline Power Consumption $(\mathrm{mW})$ & 3.55 \\
\hline Die $\operatorname{area}\left(\mu \mathrm{m}^{2}\right)$ & 127.704 \\
\hline
\end{tabular}

TABLE II. SUMMARY OF THE BANDPASS FILTER

\begin{tabular}{|l|c|c|c|c|c|}
\hline Specifications & $\begin{array}{c}{[\mathbf{1 0}]} \\
\mathbf{2 0 0 7}\end{array}$ & $\begin{array}{c}{[\mathbf{1 1}]} \\
\mathbf{2 0 0 7}\end{array}$ & $\begin{array}{c}{[\mathbf{1 2}]} \\
\mathbf{2 0 0 8}\end{array}$ & $\begin{array}{c}\text { [13] } \\
\mathbf{2 0 1 2}\end{array}$ & $\begin{array}{c}\text { This } \\
\text { work }\end{array}$ \\
\hline Technology & $\begin{array}{c}200 \mathrm{~nm} \\
\text { GaAs }\end{array}$ & $\begin{array}{c}150 \mathrm{~nm} \\
\text { GaAs }\end{array}$ & $\begin{array}{c}180 \mathrm{~nm} \\
\text { CMOS }\end{array}$ & $\begin{array}{c}180 \mathrm{~nm} \\
\text { CMOS }\end{array}$ & $\begin{array}{c}90 \mathrm{~nm} \\
\text { CMOS }\end{array}$ \\
\hline $\boldsymbol{f c}$ (GHz) & 21.98 & 22.6 & 22.7 & 23.5 & 21 \\
\hline $\begin{array}{l}\text { Power } \\
\text { dissipation } \\
\text { (mW) }\end{array}$ & 40 & 50.4 & 3.3 & 4.2 & 3.55 \\
\hline $\begin{array}{l}\text { Supply } \\
\text { voltage(V) }\end{array}$ & 3.5 & 2.1 & 1.65 & 0.7 & 1.5 \\
\hline BW(GHz) & 0.75 & 0.904 & 1.68 & 3.995 & 5.05 \\
\hline $\begin{array}{l}\text { NF (dB) } \\
\text { Die area } \\
\text { (mm }\end{array}$ & 1.95 & 17 & 14.1 & 6.7 & 3.10 \\
\hline
\end{tabular}

The layout design for the proposed bandpass filter circuit is implemented in cadence as shown in Fig. 9. The size of this layout is $10.2 \mu \mathrm{m} \times 12.52 \mu \mathrm{m}$ only. Hence it should be noted that the transistors with larger aspect ratio were designed in multifinger architecture in order to ensure required conductivity and also achieve compact core area. The FEOL corner analysis for $\mathrm{TT}, \mathrm{FF}$ and SS corners is given in Fig 10. For all the corners, the gain and noise figure remain almost stable.

Table 1 represents the performance of the proposed bandpass filter circuit and Table 2 illustrates the performance comparison with other relevant researches. From the assessment, it is evident that the proposed bandpass filter is the smallest in die area and other parameters are also very competitive. Such a fully 
integrated active bandpass filter will be very useful for K-band RF appliances.

\section{CONCLUSION}

The proposed bandpass filter circuit was evaluated through simulation by using Cadence Virtuoso. The proposed bandpass filter circuit has the center frequency of $21 \mathrm{GHz}$. Besides, it has a lower power dissipation which is $3.55 \mathrm{~mW}$ at $1.5 \mathrm{~V}$ power supply. The proposed bandpass filter circuit layout is designed by using Cadence Virtuoso GPDK 90nm CMOS technology. The size of the die area for this proposed bandpass filter circuit is equaled to $127.704 \mathrm{um}^{2}$. This indicates that the bandpass filter circuit with an active inductor design will require a much smaller area compared to the other design.

\section{REFERENCES}

[1] M. A. S. Bhuiyan, Y. Zijie, J. S. Yu, M. B. I. Reaz, N. Kamal and T. G. Chang, "Active inductor based fully integrated CMOS transmit/receive switch for $2.4 \mathrm{GHz}$ RF transceiver," Anais da Academia Brasileira de Ciências, Vol. 88, No. 2, pp. 1089-1098, 2016.

[2] M. A. S. Bhuiyan, J. X. Chew, M. B. I. Reaz and N. Kamal, "Design of an Active Inductor Based LNA in Silterra $130 \mathrm{~nm}$ CMOS process," Informacije MIDEM, Vol. 45, No. 3, pp. 188-194, 2016.

[3] M. Srivastava, D. Prasasd, and D. R. Bashkar, "New electronically tunable grounded inductor simulator employing single VDTA and one grounded capacitor," Journal of Engineering Science and Technology. Vol. 12, No. 1, pp. 113-26, 2017.

[4] S. Krishnamurthy, K. El-Sankary and E. El-Masry, "Noise-cancelling CMOS active inductor and its application in RF band-pass filter design,"
International Journal of Microwave Science and Technology. Vol. 2010, pp. 1-8, 2010.

[5] M. A. S. Bhuiyan, M. B. Omar, M. B. I. Reaz, N. Kamal and S. H. M. Ali, "A complementary metal oxide semiconductor (CMOS) bandpass filter for cost-efficient radio frequency (RF) appliances," Journal of Engg. Research. Vol. 4, pp. 114-27, 2016.

[6] R. Weng and R. Kuo, "An $\omega$ 0-Q Tunable CMOS Active Inductor for RF Bandpass Filters," International Symposium on Signals, Systems and Electronics, pp. 571-74, 2007.

[7] S. Ren and C. Benedik, "RF CMOS active inductor band pass filter with post fabrication calibration," AEU-International Journal of Electronics and Communications, Vo. 67, pp. 1058-67, 2013.

[8] L. Pantoli, V. Stornelli and G. Leuzzi, "A Low-Voltage Low-Power $0.25 \mu \mathrm{m}$ Integrated Single Transistor Active Inductor-based Filter.,"Analog Integrated Circuits and Signal Processing. Vol. 87, No. 3, pp. 463-69, 2016.

[9] M. T. I. Badal, M. B. I. Reaz, Z. Jalil, M. A. S. Bhuiyan, "Low Power High-Efficiency Shift Register Using Implicit Pulse-Triggered Flip-Flop in $130 \mathrm{~nm}$ CMOS Process for a Cryptographic RFID Tag," Electronics, Vol. 5, No. 4, No. 92, pp. 1-13, 2016. .

[10] J. Vindevoghel and P. Descamps, "Narrowband active GaAs MMIC filters in K-band," 30th European Microwave Conference, pp. 1-4, 2000.

[11] K. W. Fan, C. C. Weng, Z. M.Tsai, H. Wang and S. K.Jeng, "K-band MMIC active band-pass filters," IEEE Microw. And Wireless Compon. Lett., Vol. 15, No. 1, pp. 19- 21, 2007

[12] K. K. Huang, M. J. Chiang and C. K. C. Tzuang, "A 3.3mW K-band 0.18um 1P6M CMOS active bandpass filter using complementary currentreuse pair," IEEE Microw. and Wireless Compon. Lett., Vol. 18, No. 2, pp. 94-96, 2008.

[13] S. Wang and B. Z. Huang, "Design of low-loss and highly-selective CMOS active bandpass filter at k-band," Graduate Institute of Computer and Communication Engineering, National Taipei University of Technology, R.O.C.March,2012 